\author{
ANNALES \\ POLONICI MATHEMATICI \\ $\mathrm{XXXV}(1977)$
}

\title{
Existence theorem for functional-differential contingent equations
}

\author{
by M. KISIELEWroz and T. JANIAK (Zielona Góra)
}

\begin{abstract}
An hereditary system is a system whose present stato is determined in some way by its past history. We consider a olass of functional-differential contingent equations which involves an hereditary system. On the other hand, this class includes functional-differential contingent equations investigated by N. Kikuchi [5]. In the present paper we obtain an existence theorem for this extended olass of equa. tions.
\end{abstract}

Introduction. A number of papers have appeared recently on the subject of the optimal control of systems whose dynamies depends on the past history. These control systems can be written in terms of contingent equations. In the paper [5] N. Kikuchi has considered the control problem described by the functional-differontial contingent equation

$$
x^{\prime}(t) \in F^{\prime}\left(t, x_{t}\right)
$$

where $F$ denotes a mapping which associates with every point $\left(t, x_{t}\right)$ from a certain domain a set $F\left(t, x_{t}\right)$ in the $n$-dimensional Euclidean space, and $x_{t}(\Theta)=x(t+\Theta)$ for $\Theta \in[-r, 0]$. In this paper we shall investigate an equation which includes (1) as a particular case. The aim of our paper is to give a proof of the existence theorem for equations of such a generalized type.

1. Notations and definitions. Let $R$ denote the real line and $\boldsymbol{R}^{n}$ the real $n$-dimensional linear vector space with the norm $\|\cdot\|$. If $A$ and $B$ are compact subsets of a given metric space, then the Hausdorff metric $d(A, B)$ is defined as the smallest real number $d$ such that $A$ is contained in the $d$-neighbourhood of $B$ and $B$ in the $d$-neighbourhood of $A$. The set of all non-empty compact convex subsets of $\boldsymbol{R}^{n}$ with the Hansdorff metric is a complete metric space ([6]), which we shall denote by $\Omega^{n}$. A function $F$ defined on a real interval $[0, T]$ with values in $\Omega^{n}$ is Lebesgue measurable if for every closed subset $D \subset \boldsymbol{R}^{n}$ the set $\{t \in[0, T]: F(t) \cap D \neq \emptyset\}$ is measurable. Here, and throughout, $\emptyset$ denotes the empty sot. A function $F$ : $[0, T] \rightarrow \Omega^{n}$ is called integrably bounded if there exists a Lebesgue inte- 
grable function $h:[0, T] \rightarrow R$ such that $d(F(t),\{0\}) \leqslant h(t)$ on $[0, T]$. By $C_{n}([0, T])$ we shall denote the space of all continuous functions $x:[0, T] \rightarrow R$ with the supremum norm $\|x\|_{c}=\max \{\|x(t)\|: t \in[0, T]\}$ and by $L_{n}([0, T])$ the space of all Lebesgue summable functions from $[0, T]$ to $\boldsymbol{R}^{n}$ with the norm $\|x\|_{l}=\int_{0}^{T}\|x(t)\| d t$. The space of all real valued functions with summable $p$-th power we shall denote by $L^{2}([0, T])$. In this case we put $\|h\|_{L^{2 p}} \doteq\left(\int_{0}^{T^{1}}|\hbar(t)|^{p} d t\right)^{1 / p}$ for $h \in L^{p}([0, T]) ; p \geqslant 1$.

Let $A$ denote the set of all non-empty compact subset of $R$ and let $A$ be an element of $A$. It is convenient to assume that zero is the maximal element of $A$. Let $C_{A}=O_{12}(A)$. Suppose that $E$ is a interval of $R$ and $\alpha$ :

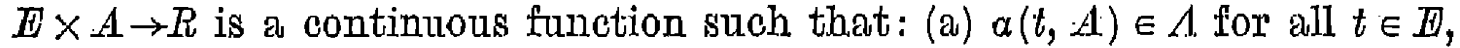
(b) $\alpha(t, \Theta) \leqslant t$ for $t \in E$ and $\Theta \in A,(c) \alpha(t, \Theta) \leqslant \alpha(t, \eta)$ for $t \in E$ and $\Theta<\eta$,

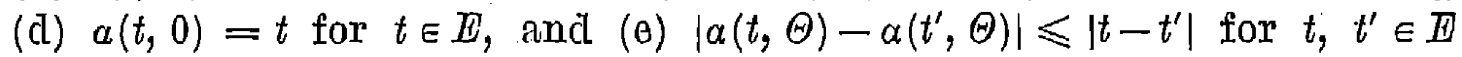
and $\Theta \in A$. Let ue denote by $X$ the space of all continuous functions $x$ : $P \rightarrow \boldsymbol{R}^{n}$, where $P=a(E \times A)$. For every fixed $t \in E$ the mapping $H_{t}$ : $X \rightarrow O_{\Delta}$ will be defined by $\left(H_{t} x\right)(\Theta)=x[\alpha(t, \Theta)]$ for $\Theta \in A$. The triple $(A, a, H)$ will be called as an hereditary structure. By substructure of $(A, \alpha, H)$ we shall mean the triplo $(\bar{A}, \bar{\alpha}, \bar{H})$, where $\bar{A} \in \Lambda$ and $\bar{A} \subset A$, $\bar{a}=a \mid \bar{A}$ and $\bar{H}$ is defined by $\left(\bar{H}_{t} x\right)(\Theta)=x[\bar{\alpha}(t, \Theta)]$.

Let $(A, \alpha, H)$ be an hereditary structure and let $g: E \times C_{\Lambda} \rightarrow \boldsymbol{R}^{n}$ and $F$ : $E \times C_{A} \rightarrow \Omega^{n}$. In this paper we shall investigate a functional-differential contingent equation of the form

$$
\frac{d}{d t}\left[D(t) H_{t} x\right] \in F\left(t, H_{t} x\right),
$$

where $D(t) \Phi=\Phi(0)-\dot{g}(t, \Phi)$ for $t \in \mathbb{E}$ and $\Phi \in C_{A}$.

To appreciate the generality of (2), let us consider some special cases of this equation. If $g=0$, then (2) reduces to the equation

$$
x^{\prime}(t) \in F\left(t, H_{t} x\right) \text {. }
$$

If $A=[-r, 0], r \geqslant 0, \alpha(t, \Theta)=t+\Theta, \Theta \in[-r, 0],\left(H_{t} x\right)(\Theta)=x(t+\Theta)$, $\Theta \in[-r, 0]$, then (3) reduces to the functional-differential contingent equations of retarded type

$$
x^{\prime}(t) \in F\left(t, x_{t}\right)
$$

where we employed the conventional notation $x_{t}(\Theta)=x(t+\Theta)$. Preserving: this simple notation, we see that (2) includes the general class of equations

$$
\frac{d}{d t}\left[D(t) x_{t}\right] \in F^{\prime}\left(t, x_{l}\right)
$$


If $F=\{f\}$, where $f:[0, T] \times C_{A} \rightarrow \boldsymbol{R}^{n}$, then (2) has the form

$$
\frac{d}{d t}\left[D(t) \dot{H}_{t} x\right]=f\left(t, H_{t} x\right),
$$

which has been first investigated by Hale and Cruz ([3]).

We now formulate the initial value problem for (2). For a hereditary structure $(A, \alpha, B)$ and $\sigma \in E$ let $E_{\sigma}$ be the set of real numbers defined by

$$
E_{\sigma}=\bigcup_{\substack{t \geq \sigma \\ i \in E}} \overline{\operatorname{co}} \alpha(t, A) \cap(-\infty, \sigma],
$$

where by $\overline{c o} G$ we mean the closed convex hull of $G$. Given a $\sigma$ in $E$ and a function $\Phi \in C_{A}$, we say that $\infty=\infty(\sigma, \Phi)$ is a solution of (2) with the initial function $\Phi$ at $\sigma$, if there is a $\gamma>0$ such that $x$ is defined and continuous on $E_{\sigma} \cup[\sigma, \sigma+\gamma], x$ coincides with $\Phi$ on $\mathbb{H}_{\sigma}, D(t) H_{t} x$ is absolutely continuous on $[\sigma, \sigma+\gamma]$ and satisfies (2) on $[\sigma, \sigma+\gamma]$.

2. Fixed point theorem. The analogue of the Schauder fixed point theorem, which we shall need in this section, is due to Bohenenblust and Karlin in [1]. In the formulation most readily useful for our purpose this states.

THEOREM 1. Let $S$ be a compact convex subset of a Banach space and let $I$ a continuous (in the Hausdorff topology) mapping of $S$ into the space of non-empty closed convex subsets of S. Then $\Gamma$ has a fixed point, i.e., there exists $x \in \mathbb{S}$, such that $x \in \Gamma(x)$.

In this section we give a slight generalization of this type fixed point theorem. Let $X$ be a Banach space and let us denote by Conv $(X)$ the collection of all non-empty closed convex subsets of $X$. We, shall prove the following lemma.

Lemara 2. Let $S$ be a compact convex subset of $X$ and let $T: X \rightarrow X$ be a linear mapping such that $|T| \leqslant \lambda<1$. Suppose that $\Gamma: S \rightarrow \operatorname{Oon} \nabla(X)$ is continuous and such that $T(S)+\Gamma(x) \subset S$ for every $x \in S$. Then $T+\Gamma$ has a fixed point.

- Proof. Let $I$ be the identity mapping. Since $T$ is a contraction, then $I-T$ is a homeomorphism between $S$ and $(I-T)(S)$. We shall show that $\Gamma(x) \subset(I-T)(S)$ for every $x \in S$. Suppose $y \in \Gamma(x)$ for fixed $x \in S$. Let $\left\{x_{n}\right\}$ will be defined in the following way: $x_{0} \in S, x_{n+1}=y+T\left(x_{n}\right)$ for $n=0,1,2, \ldots$ We have $x_{1} \in T\left(x_{0}\right)+\Gamma(x) \subset T(S)+\Gamma(x) \subset S$. In a similar way we obtain $x_{n} \in S$ for $n=2,3, \ldots$ Furthermore, we have $\left\|x_{n+1}-x_{n}\right\|_{X}=\left\|T\left(x_{n}\right)-T\left(x_{n-1}\right)\right\|_{X} \leqslant|T|\left\|x_{n}-x_{n-1}\right\|_{X} \leqslant \lambda\left\|x_{n}-x_{n-1}\right\|_{X}$ for $n$ $=1,2, \ldots$, where $\lambda \in(0,1)$. Therefore $\left\{x_{n}\right\}$ forms a Cauchy sequence which must converge to an element $\tilde{x} \in S$. It is clear that $\tilde{x}$ satisfies $(I-T)(\tilde{x})=y$. Oonsoquently, for every $\bar{y} \in \Gamma(x)$ there is a $\tilde{x} \in S$ such 
that $y=(I-T)(\tilde{x}) \in(I-T)(S)$. Thus for every $x \in S$ we have $\Gamma(x)$ C $(I-T)(S)$.

Let us observe that finding a fixed point of $T+T$ in $S$ is equivalent to finding a fixed point of $(I-T)^{-1} \circ \Gamma$ defined by $\left[(I-T)^{-1} \circ \Gamma\right](x)$ $=(I-T)^{-1}(\Gamma(x))$ for $x \in S$. Indeed, let $x \in S^{\prime}$ be a fixed point of $(I-T)^{-1} \circ \Gamma$, i.e., let $x \in\left[(I-T)^{-1} \circ \Gamma\right](x)$. There exists an element $y \in \Gamma(x)$ such that $x=(I-T)^{-1}(y)$. Therefore $x=T(x)+y$, i.e., $x \in(T+\Gamma)(x)$. Suppose that $x \in\left(T+I^{\prime}\right)(x) \cap S$. Then $x-T(x) \in \Gamma(x)$. Therefore there is an element $y \in \Gamma(x)$ such that $x-T(x)=y$, i.e., $x=(I-T)^{-1}(y)$. Consequently $x \in\left[(I-T)^{-1} \circ I\right](x)$.

Now we shall show that $(I-T)^{-1} \circ \Gamma$ satisfies the hypothesis of Theorem 1. By Proposition 4.3 given in [4], the mapping $(I-T)^{-1} \circ \Gamma$ is continuous on $S$. From $\Gamma(x) \subset(I-T)(S)$ it follows that $\left[(I-T)^{-1} \circ \Gamma\right](x) \subset S$ for every $x \in S$. Obviously, $\left[(I-T)^{-1} \circ T\right](x)$ is closed for every $x \in S$. To see that $\left[(I-T)^{-1} \circ I\right](x)$ is convex set, suppose $u, v \in\left[(I-T)^{-1} \circ \Gamma\right](x)$ for fixed $x \in S$. Then there are $z, w \in \Gamma(x)$ such that $u=z+T(u)$ and $v=w+T(v)$. Since $v u+(1-v) v=v z+(1-v) w+$ $+T(\nu u+(1-v) v)$ and $\nu z+(1-v) w \in \Gamma(x)$ for $v \in[0,1]$, then there is an element $y \in \Gamma(x)$ such that $v u+(1-v) v=(I-T)^{-1}(y)$. Therefore $v u+$ $+(1-v) v \in\left[(I-T)^{-1} \circ \Gamma\right](x)$ for $x \in S$ and $v \in[0,1]$. Hence, in virtue of Theorem 1 , the mapping $(I-T)^{-1} \circ \Gamma$ has a fixed point. This completes the proof.

3. Existence of solutions of (2). In this section we write $I=[0, T]$ ) For given function $G: I \rightarrow \Omega^{n}$ by $\mathscr{F}_{I}(G)$ we shall denote the set of all Le. besgue integrable functions $u: I \rightarrow \boldsymbol{R}^{n}$ having the property that $u(t) \in G(t$ for almost every $t \in I$. Let $\int_{0}^{t} G(\tau) d \tau=\left\{\int_{0}^{t} u(\tau) d \tau: u \in \mathscr{F}_{I}(G)\right\}$ for $t \in I$. It has been proved in [2] that for every measurable and integrably bounded mapping $G: I \rightarrow \Omega^{n}$. We have $\mathscr{F}_{I}(G) \neq \varnothing$ and that $\int_{0}^{t} G(\tau) d \tau$ is a compact, convex subset of $C_{n}(I)$.

We will say that a mapping $F: I \times C_{A} \rightarrow \Omega^{n}$ satisfies the Carathéodory type conditions if $F(t, \psi)$ is measurable in $t \in I$ for fixed $\psi \in C_{A}, F(t, \psi)$ is continuous in $\psi \in O_{A}$ for fixed $t \in I$ and there exists $m \in L^{2}(I)$ such that $a(F(t, \psi),\{0\}) \leqslant m(t)$ for $(t, \psi) \in I \times C_{A}$.

We shall need the following lemma.

Lemax 3. Suppose that $(A, \alpha, H)$ is an hereditary struoture and that $I$ : $I \times O_{A} \rightarrow \Omega^{n}$ satisfies the Caratheodory type conditions. Then for every $x \in C_{n}(A \cup I)$ the mapping $G: I \rightarrow \Omega^{n}$ defined by $G(t)=F\left(t, H_{t} x\right)$ is measwrable and integrably bounded.

Proof. From the definition of $G$ it follows that $d(G(t),\{0\}) \leqslant m(t)$ for $t \in I$. It has been proved in [2] (see Lemma 2.8) that if $\Phi: I \times D \rightarrow \Omega^{n}$, 
where $D \subset \boldsymbol{R}^{n}$, satisfies the Carathédory type conditions, then for every $x \in C_{n}(I)$ such that $x(I) \subset D$, the mapping $\Phi(t, x(t))$ is measurable on $I$. Let us write $\Phi(t, z)=F\left(t, H_{z} x\right)$ for fixed $x \in C_{n}(I \times A)$ and $(t, z)^{-} \in I \times I$. It is clear that $\Phi(t, z)$ satisfies tho Carathéodory type conditions. Therefor $\theta$ the mapping $\Phi(t, x(t))$ is measurable on $I$ for every continuous function $x$ : $I \rightarrow I$. Taking $x(t)=t$ we get the measurability of $G$ on $I$. This completes. the proof.

Let $\sigma \in I$ and let us denote by $I_{\delta}(\sigma)$ the interval $[\sigma, \sigma+\delta]$ for fixed $\delta>0$. For a given $\Phi \in C_{E}$ we define function $\tilde{\Phi}$ on $E_{\sigma} \cup I_{\delta}(\sigma)$ as follows

$$
\tilde{\Phi}(t)= \begin{cases}\Phi(t) & \text { for } t \in E_{\sigma}, \\ \Phi(\sigma) & \text { for } t \in I_{\mathrm{d}}(\sigma) .\end{cases}
$$

Assume that $g: I \times C_{A} \rightarrow \boldsymbol{R}^{n}$ is a mapping satisfying the following conditions: (i) $g$ is continuous on $I \times C_{A}$, (ii) $g$ is linear with respect to $\psi \in C_{A}$ and such that there is a number $\lambda_{g} \in(0,1)$ so that $\sup \left\{\|g(t, \psi)\|:\|\psi\|_{c}=1\right\} \leqslant \lambda_{o}$ for $t \in I$.

We prove the following lemma.

LEMMA 4. Suppose that $(A, a, H)$ is an hereditary structure, $g$ satisfies (i)-(ii), and let $\lambda \in\left(\lambda_{g}, 1\right)$. Then there are $\gamma>0$ and a hereditary substructure $(\bar{A}, \tilde{\sigma}, \bar{H})$ of $(A, a, H)$ such that $\left\|g\left(t, \bar{H}_{t} \tilde{\Phi}\right)-g\left(\sigma, \bar{H}_{\sigma} \tilde{\Phi}\right)\right\| \leqslant \lambda-\lambda_{0}$ for every $t \in I_{\gamma}(\sigma)$ and $\Phi \in C_{A}$.

Proof. For $t \in I$ we have

$$
\begin{aligned}
\left\|g\left(t, H_{t} \tilde{\Phi}\right)-g\left(\sigma, H_{\sigma} \tilde{\Phi}\right)\right\| \leqslant \| g\left(t, H_{t} \tilde{\Phi}\right)- & g\left(\sigma, H_{t} \tilde{\Phi}\right) \|+ \\
& +\left\|g\left(\sigma, H_{t} \tilde{\Phi}\right)-g\left(\sigma, H_{\sigma} \tilde{\Phi}\right)\right\| .
\end{aligned}
$$

By continuity of $g$ there exists $\gamma>0$ such that

$$
\left\|g\left(t, H_{t} \tilde{\Phi}\right)-g\left(\sigma, H_{t} \tilde{\Phi}\right)\right\| \leqslant \frac{1}{2}\left(\lambda-\lambda_{g}\right) \quad \text { for } t \in I_{\gamma}(\sigma) \text {. }
$$

Furthermore; we have

$$
\left\|\dot{g}\left(\sigma, H_{t} \tilde{\Phi}\right)-g\left(\sigma, H_{\sigma} \tilde{\Phi}\right)\right\| \leqslant \lambda_{g}\left\|H_{t} \tilde{\Phi}-H_{\sigma} \tilde{\Phi}\right\|_{c} .
$$

Since $\left\|H_{i} \tilde{\Phi}-H_{\sigma} \tilde{\Phi}_{\|_{c}}=\sup _{\theta \in A}\right\| \Phi(\alpha(t, \Theta))-\Phi(\alpha(\sigma, \Theta)) \|$ for $\alpha(t, \Theta) \in E_{\sigma}$ and $\left\|H_{t} \tilde{\Phi}-\dot{H}_{\sigma} \tilde{\Phi}_{\|_{\sigma}}=\sup _{\Theta \in A}\right\| \Phi(\alpha(\sigma, 0))-\Phi(\alpha(\sigma, \Theta)) \|$ for $\alpha(t, \Theta) \in I_{\gamma}(\sigma)$; then it. follows that

$$
\left\|H_{t} \tilde{\Phi}-H_{\sigma} \tilde{\Phi}\right\|_{c} \leqslant \frac{\lambda-\lambda_{g}}{2 \lambda_{g}}
$$

whenever $\gamma$ and $\operatorname{diam}(A)$ are sufficiently small. This completes the proof..

Now we can prove the existence of solution of (2).

THEOREM 5. Suppose that $(A, a, H)$ is an hereditary structure, $F$ : $I \times C_{A} \rightarrow \Omega^{n}$ satisfies the Carathéodory type condition $g: I \times O_{A} \rightarrow \boldsymbol{R}^{n 2}$ satisfies assumptions of Lemma $4, \sigma \in I$ and $\Phi \in C_{A}$. Then there are substructure: 
$(\bar{A}, \bar{a}, \bar{H})$ of $(A, a, H)$ and $a \gamma>0$ such that (2) with the initial function $\Phi$ at $\sigma$ corresponding to $(\bar{A}, \bar{a}, \bar{H})$ has at least one solution $x(\Phi, \sigma)$ defined on $E_{\sigma} \cup I_{\gamma}(\sigma)$.

Pro of. Suppose that $\gamma>0$ and $(\bar{A}, \bar{\alpha}, \bar{H})$ are such as in Lemma 4. It will be convenient to write $(A, \alpha, H)$ instead of $(\bar{A}, \bar{\alpha}, \bar{H})$. Let $I_{\gamma}=I_{\gamma}(\sigma)$ and let $\omega_{g}(\varepsilon)=\sup \left\{\left\|g(t, \psi)-g\left(t^{\prime}, \psi^{\prime}\right)\right\|: t, t^{\prime} \in I, \psi, \psi^{\prime} \in C_{A},\left|t-t^{\prime}\right| \leqslant \varepsilon\right.$, $\left.\left\|\psi-\psi^{\prime}\right\|_{\varepsilon} \leqslant \varepsilon\right\}$ and $\omega_{\Phi}(\varepsilon)=\sup \left\{\left\|\Phi(t)-\Phi\left(t^{\prime}\right)\right\|: t, t^{\prime} \in A,\left|t-t^{\prime}\right| \leqslant \varepsilon\right\}$ for $\varepsilon \geqslant 0$. Consider the set $S \subset C_{n}\left(E_{\sigma} \cup I_{\gamma}\right)$ of all continuous functions $x$ : $E_{\sigma} \cup Y_{\gamma} \rightarrow \boldsymbol{R}^{n}$ such that $x(t)=0$ for $t \in E_{a},\left\|x(t)-\infty\left(t^{\prime}\right)\right\| \leqslant \varrho\left(\left|t-t^{\prime}\right|\right)$ for $t, t^{\prime} \in[\sigma, T],\|x\|_{e} \leqslant M$, where

$$
\varrho(\varepsilon)=\frac{\omega_{\theta}(\varepsilon)+\lambda_{\theta} \omega_{\Phi}(\varepsilon)+\|m\|_{L^{2}} \sqrt{\varepsilon}}{1-\lambda_{\theta}} \text { and } M=\frac{1+\|m\|_{L^{2}}}{1-\lambda_{g}} .
$$

It is easy to see that $\dot{S}$ is a compact convex subset of $O_{n}\left(E_{\sigma} \cup I_{\gamma}\right)$. For every $x \in S$ let $Y(x)=\mathscr{F}_{I}\left[F\left(t, H_{t}(x+\Phi)\right)\right]$ for fixed $t \in I$ and let

$(\Gamma x) .(t)= \begin{cases}\{0\} & \text { for } t \in E_{\sigma}, \\ {\left[g\left(t, H_{t} \tilde{\Phi}\right)-g\left(\sigma, H_{\sigma} \tilde{\Phi}\right)\right]+\int_{\sigma}^{t} F\left(\tau, H_{\tau}(x+\tilde{\Phi})\right) d \tau} & \text { for } t \in I_{\gamma} .\end{cases}$

In virtue of Lemma 3 and by the properties of $\int_{0}^{t} F\left(\tau, H_{\tau}(x+\tilde{\Phi})\right) d \tau$ (see [2]) we can see that $\Gamma x \in \operatorname{Comp}\left(C_{n}\left(E_{o} \cup I_{\gamma}\right)\right)$ for overy $\infty \in S$. Then $\Gamma: \$ \rightarrow$ $\operatorname{Comp}\left(C_{n}\left(E_{\sigma} \cup I_{\gamma}\right)\right)$. To see that $\Gamma$ is continuous on $S$ let $x_{0}$ be an arbitrary element of $S$ and let $\left\{x_{n}\right\}$ be any sequence of $S$ such that $\left\|x_{n}-x_{0}\right\|_{0} \rightarrow 0$ as $n \rightarrow \infty$. Since

$$
\begin{aligned}
\left\|H_{l}\left(x_{n}+\tilde{\Phi}\right)-H_{t}\left(x_{0}+\tilde{\Phi}\right)\right\|_{0} & =\sup _{\Theta \in \dot{A}} \| x_{n}\left[\alpha(t, \Theta)-x_{0}[\alpha(t, \Theta)] \|\right. \\
& \leqslant \sup _{\Theta \in A}\left\{\sup _{\sigma \leqslant u \leqslant a(t, \Theta)}\left\|x_{n}(u)-x_{0}(u)\right\|\right\} \\
& \leqslant \sup _{\sigma \leqslant u \leqslant t}\left\|x_{n}(u)-x_{0}(u)\right\| \leqslant\left\|x_{n}-x_{0}\right\|_{c},
\end{aligned}
$$

then

$$
\left\|H_{t}\left(x_{n}+\tilde{\Phi}\right)-H_{t}\left(x_{0}+\tilde{\Phi}\right)\right\|_{0} \rightarrow 0 \quad \text { whenever }\left\|x_{n}-x_{0}\right\|_{o} \rightarrow 0 \text {. }
$$

Therefore

$$
\lim _{n \rightarrow \infty} d\left[\not F\left(t, H_{t}\left(x_{n}+\tilde{\Phi}\right)\right), F\left(t, H_{t}\left(x_{0}+\tilde{\Phi}\right)\right)\right]=0 \quad \text { for } t \in I .
$$

Hence, in virtue of Theorem 3.2 given in [2], wo get

$$
\lim _{n \rightarrow \infty} h\left[\int_{\sigma}^{t} F\left(\tau, H_{\tau}\left(x_{n}+\tilde{\Phi}\right)\right) d \tau, \int_{\sigma}^{t} F\left(\tau, H_{\tau}\left(x_{0}+\tilde{\Phi}\right)\right) d \tau\right]=0,
$$

where $h$ denotes the Hausdorff metric in $\operatorname{Comp}\left(C_{n}\left(E_{\sigma} \cup I_{\gamma}\right)\right)$. Therefore we have $\lim _{n \rightarrow \infty} h\left(\Gamma x_{n}, \Gamma x_{0}\right)=0$. 
Let $T: C_{n}\left(E_{\sigma} \cup I_{\gamma}\right) \rightarrow C_{n}\left(E_{\sigma} \cup I_{\gamma}\right)$ be a mapping defined by

$$
(T x)(t)= \begin{cases}0 & \text { for } t \in E_{\sigma}, \\ g\left(t, H_{t} x\right) & \text { for } t \in I_{\nu}\end{cases}
$$

It is easy to see that $T$ is a linear mapping. Furthermore we have

$$
\begin{aligned}
|T|=\sup _{x}\left\{\|T x\|_{\boldsymbol{c}}:\|x\|_{c}=1\right\} & \leqslant \sup _{x}\left\{\sup _{t \in I}\left\|g\left(t, H_{t} x\right)\right\|:\|x\|_{e}=1\right\} \\
& \leqslant \sup _{x}\left\{\lambda_{g} \sup _{t \in I}\left\|H_{t} x\right\|:\|x\|_{e}=1\right\}=\lambda_{g}<1 .
\end{aligned}
$$

For every $y \in S, x \in S, z \in T y+\Gamma x$ and $t \in I_{\gamma}$ we have

$$
\begin{aligned}
\|z(t)\| \leqslant\left\|g\left(t, H_{t}(y+\tilde{\Phi})-g\left(t, H_{t} \Phi\right)\right)\right\|+\left\|g\left(t, \dot{H}_{t} \tilde{\Phi}\right)-g\left(\sigma, H_{\sigma} \tilde{\Phi}\right)\right\|+ & +\int_{\sigma}^{t} m(\tau) d \tau \\
& \leqslant \lambda_{g}\left\|H_{t} y\right\|_{\mathbf{c}}+\left(\lambda-\lambda_{g}\right)+\|m\|_{L^{2}} \leqslant \lambda_{g}\|y\|_{c}+1+\|m\|_{L^{2}} \\
& \leqslant \lambda_{g} M+1+\|m\|_{L^{2}},
\end{aligned}
$$

where $M=\frac{1+\|m\|_{L^{2}}}{1-\lambda_{g}}$. Then $\|z\|_{c} \leqslant M$ for every $y \in S$ and $x \in S$. For $t>t^{\prime} \in I_{\gamma}$ and $z \in T y+\Gamma x$ we obtain

$$
\begin{aligned}
\left\|z(t)-z\left(t^{\prime}\right)\right\| \leqslant & \left\|g\left(t, H_{l}(y+\tilde{\Phi})\right)-g\left(t^{\prime}, H_{l}(y+\Phi)\right)\right\|+ \\
& +\| g\left(t^{\prime}, H_{l}(y+\Phi)\right)-g\left(t^{\prime}, H_{l^{\prime}}(y+\tilde{\Phi}) ; \|+\int_{t^{\prime}}^{t} m(\tau) d \tau\right. \\
\leqslant & \omega_{g}\left(\left|t-t^{\prime}\right|\right)+\lambda_{g}\left\|H_{t} y-H_{t^{\prime}} y\right\|_{c}+\lambda_{g}\left\|H_{t} \tilde{\Phi}-H_{l^{\prime}} \tilde{\Phi}\right\|_{c}+\|m\|_{L^{2}} \sqrt{\left|t-t^{\prime}\right|} \\
\leqslant & \omega_{g}\left(\left|t-t^{\prime}\right|\right)+\|m\|_{L^{2}} \sqrt{\left|t-t^{\prime}\right|}+\lambda_{g} \omega_{g}\left(\left|t-t^{\prime}\right|\right)+\lambda_{g}\left\|H_{l} y-H_{t^{\prime}} y\right\|_{c} .
\end{aligned}
$$

Since

$$
\left\|H_{\iota} y-H_{t^{\prime}} y\right\|=\|y(\alpha(t, \Theta))-y(\alpha(t, \Theta))\| \leqslant \varrho\left(\mid \alpha(t, \Theta)-\alpha\left(t^{\prime}, \Theta\right) \| \varrho\left(\left|t-t^{\prime}\right|\right),\right.
$$

then, by the definition of the set $S$, we get

$$
\left\|z(t)-z\left(t^{\prime}\right)\right\| \leqslant \varrho\left(\left|t-t^{\prime}\right|\right) \quad \text { for } t>t_{j}^{\prime} \in I_{\gamma} .
$$

Consequently $z \in S$. Therefore $T(S)+\Gamma x \subset S$ for every $x \in S$. Hence, in virtue of Lemma 2 , follows the existence of $\tilde{x} \in S$ such that $\tilde{x} \in(T+\Gamma)(\tilde{x})$.

Now we shall show that $\tilde{y}=\tilde{x}+\tilde{\Phi}$ is a solution of (2) with initial function $\Phi$ at $\sigma$. By the definition of $T$ and $\Gamma$ it follows that $\tilde{x}(t)=0$ for $t \in E_{\sigma}$; then $\tilde{y}(t)=\Phi(t)$ on $\mathbb{E}_{\sigma}$. On the other hand, from $\tilde{w} \in T \tilde{x}+\Gamma \tilde{x}$ for $t \in I_{\gamma}$ it follows that

$$
\tilde{x}(t)=g\left(t, H_{t}(\tilde{x}+\tilde{\Phi})\right)-g\left(\sigma, H_{0} \tilde{\Phi}\right)+\int_{0}^{t} u(\tau) d \tau,
$$


where $u(\tau) \in F\left(\tau, H_{\tau}(\bar{x}+\tilde{\Phi})\right)$ for a.e. $t \in I_{\gamma}$. Hence

$$
D(t) H_{l}(\tilde{a}+\tilde{\Phi})-D(\sigma) \dot{H}_{\sigma}(\tilde{x}+\tilde{\Phi})=\int_{\sigma}^{t} u(\tau) d \tau .
$$

Therefore twe have .

$$
\frac{d}{d t}\left[D(t) H_{t} \tilde{y}\right] \in F\left(t, H_{t} \tilde{y}\right) \quad \text { for a.e. } t \in I_{\gamma} \text {. }
$$

This completes the proof.

\section{References}

[1] H. F. Bohenonblust and S. Karlin, On a theorem of Ville, Ann. Math. Studies, No. 24, p. 155-160. Princeton, N.J. (1950).

[2] T. F. Bridgland, Js., Trajectory integrals of set valued funotions, Pacific J.' Math. 33 (1) (1970), p. 43-68.

[3] J. K. Hale and M. A. Cruz, Existence uniqueness and continuous dependenoe for hereditary systems, Ann. Mat. Pur'a Appl. 85 (1970), p. 63-81.

[4] M. Huku haxy, Sur l'application semisontinue dont la valuer est un compact convexe, Funk, Elkv. 10 (1967), p. 44-66.

[5] N. Kikuchi, On control problems for functional-differential equations, ibidem 14 (1971), p. 1-23.

[6] E. Michael, Topologies on spaoes of subsets, Trans. Amer. Math. Soc. 71 (1951), p. 152-182. 